\title{
The Movement Tracker: A Flexible System for Automated Movement Analysis in Invertebrate Model Organisms
}

\author{
Laurent Mouchiroud, ${ }^{1,4}$ Vincenzo Sorrentino, ${ }^{1,4}$ Evan G. Williams, ${ }^{1}$ \\ Matteo Cornaglia, ${ }^{2}$ Michael V. Frochaux, ${ }^{3}$ Tao Lin, ${ }^{1}$ \\ Amandine A. Nicolet-dit-Félix, ${ }^{1}$ Gopal Krishnamani, ${ }^{1}$ Tarik Ouhmad, ${ }^{1}$ \\ Martin A.M. Gijs, ${ }^{2}$ Bart Deplancke, ${ }^{3}$ and Johan Auwerx ${ }^{1}$

\footnotetext{
${ }^{1}$ Laboratory of Integrative and Systems Physiology, École Polytechnique Fédérale de Lausanne, Lausanne, Switzerland

${ }^{2}$ Laboratory of Microsystems, École Polytechnique Fédérale de Lausanne, Switzerland, Lausanne, Switzerland

${ }^{3}$ Laboratory of Systems Biology and Genetics, École Polytechnique Fédérale de Lausanne, Switzerland, Lausanne, Switzerland
} \\ ${ }^{4}$ These authors contributed equally to this work.
}

Phenotyping strategies in simple model organisms such as D. melanogaster and C. elegans are often broadly limited to growth, aging, and fitness. Recently, a number of physical setups and video tracking software suites have been developed to allow for accurate, quantitative, and high-throughput analysis of movement in flies and worms. However, many of these systems require precise experimental setups and/or fixed recording formats. We report here an update to the Parallel Worm Tracker software, which we termed the Movement Tracker. The Movement Tracker allows variable experimental setups to provide crossplatform automated processing of a variety of movement characteristics in both worms and flies and permits the use of simple physical setups that can be readily implemented in any laboratory. This software allows high-throughput processing capabilities and high levels of flexibility in video analysis, providing quantitative movement data on C. elegans and D. melanogaster in a variety of different conditions. (C) 2016 by John Wiley \& Sons, Inc.

Keywords: model organisms - software - movement - neurodegeneration $\bullet$ aging $\bullet$ C. elegans $\bullet$ D. melanogaster

\section{How to cite this article:}

Mouchiroud, L., Sorrentino, V., Williams, E.G., Cornaglia, M., Frochaux, M.V., Lin, T., Nicolet-dit-Félix, A.A., Krishnamani, G., Ouhmad, T., Gijs, M.A.M., Deplancke, B., and Auwerx, J. 2016. The movement tracker: A flexible system for automated movement analysis in invertebrate model organisms. Curr. Protoc. Neurosci.

77:8.37.1-8.37.21. doi: $10.1002 /$ cpns. 17

\section{INTRODUCTION}

Given the relative ease of studying C. elegans and Drosophila, these organisms have long been ubiquitous in studies on basic genetics and for mechanistic validation of molecular pathways. Until recently, due to the difficulty and expense in acquiring highquality digital imaging and video, assays on the movement activity of these organisms were often done qualitatively. In the past decade, such recordings have become trivial 
to obtain, yet unlike with increasingly standardized mouse phenotyping protocols (e.g., EMPReSS), the invertebrate tracking systems have diversified such that reviews have been published to track the tracking software (Husson et al., 2012). While a simple, standardized (yet adaptable) software suite is unlikely to be suitable for the diversity of research on C. elegans and Drosophila, we provide these protocols and software as a cornerstone towards a unification and standardization of movement tracking procedures using different model organisms (e.g., worm models of neurodegeneration) and a variety of experimental platforms.

BASIC PROTOCOL

\section{EXPERIMENTAL DESIGN FOR MOVEMENT TRACKING WITH C. elegans USING THE MOVEMENT TRACKER}

As locomotor activity is a highly complex phenotype dependent on numerous genetic and environmental interaction effects, different assays may yield substantially different results. Furthermore, subtle changes in time of day, temperature, age, and sex can be difficult to control yet have significant impact on the results obtained. We have used three experimental conditions, based on differences in environment or model organism used, for validating the consistency of movement tracking (Fig. 8.37.1A-C). For the nematode C. elegans, we have tested the Movement Tracker with two different environments: solid medium (nematode growth medium [NGM] plates; Basic Protocol; Fig. 8.37.1A) and

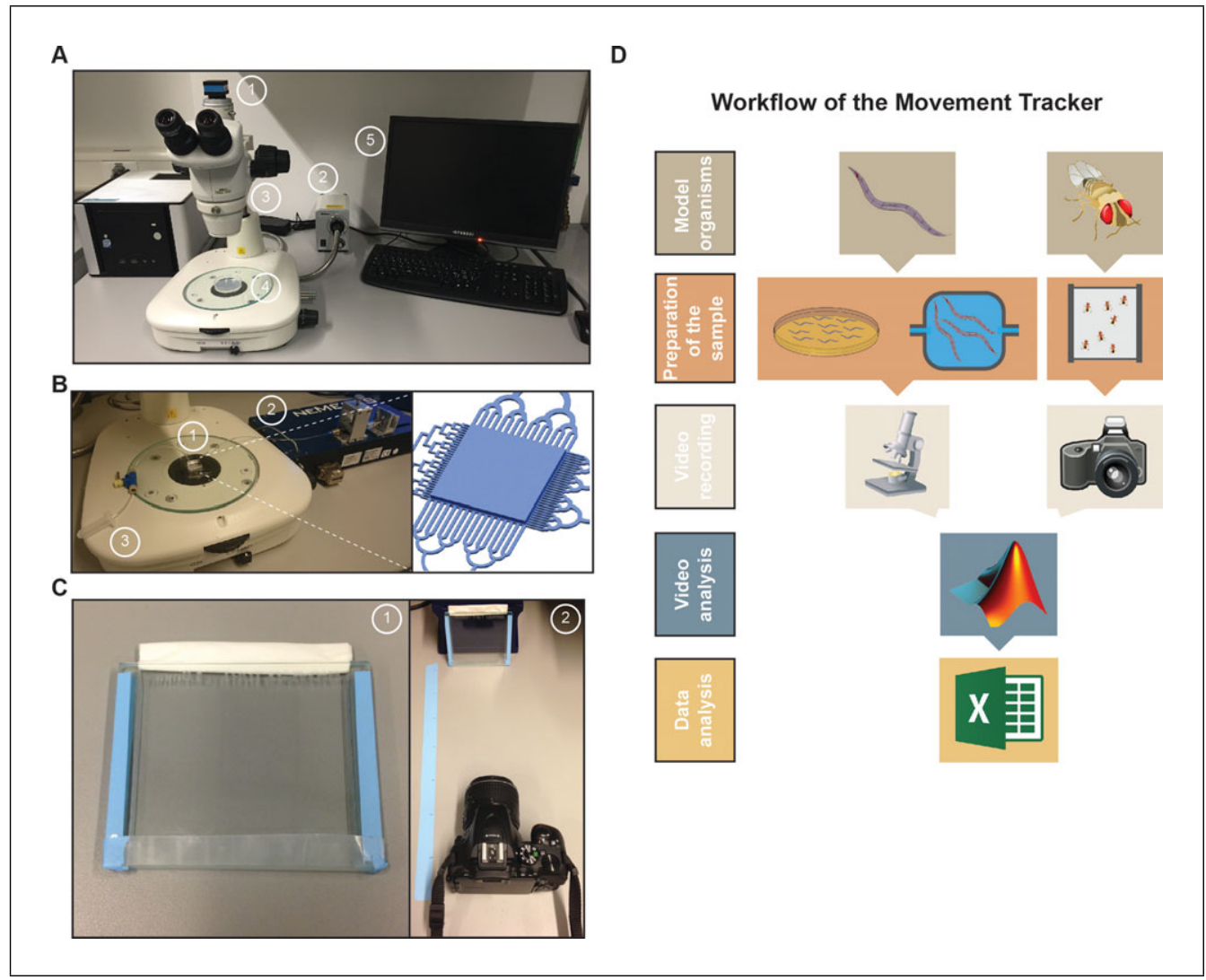

Figure 8.37.1 Experimental setup of the Movement Tracker. (A) Image of the Movement Tracker setup for $C$. elegans on solid medium, including (1) a camera, (2) a light source, (3) a stereomicroscope, (4) the sample, and (5) a computer. (B) Image of the Movement Tracker setup for

Automated Quantitative

Analysis of Movement in Invertebrate Models C. elegans on a microfluidic chip device, including the light source, a stereomicroscope, (1) the microfluidic chip and its design, (2) the injection system including pumps and syringes, and (3) a waste container. (C) Image of the Movement Tracker setup for Drosophila, including (1) the pad and (2) the experimental setup composed of the pad, the camera, and a marking tape to measure distance. (D) Schematic representation of workflow for the different setups of the Movement Tracker. 
A

Worms on NGM plates

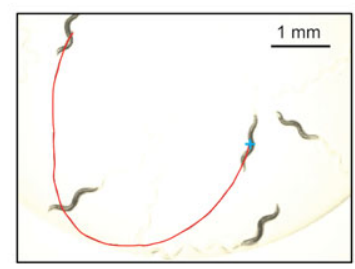

D

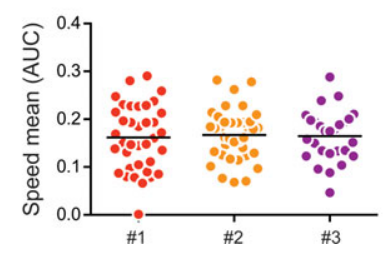

Worms in microfluidic

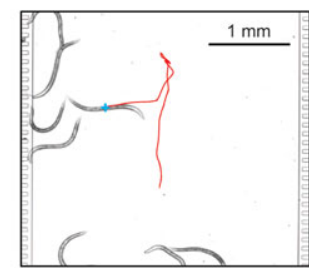

$E$

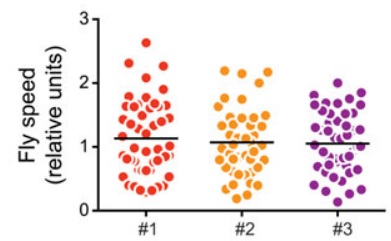

C

Flies in basal movement

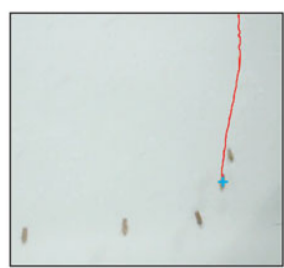

Figure 8.37.2 Overview of the data obtained with the Movement Tracker in different setups. Representative pictures of individual tracks obtained with the Movement Tracker for worms on solid agar medium (A), in a microfluidic chip (B), and for Drosophila (C). Movement of wild-type worms at day 1 of adulthood (D) and Drosophila at day 1 of adulthood (E) in three independent experiments. Each single dot represents the movement of a single individual. AUC, area under the curve.

liquid (microfluidics system; Alternate Protocol 1; Fig. 8.37.1B). For D. melanogaster we have measured spontaneous walking movement (Alternate Protocol 2; Fig. 8.37.1C). In each case, we have observed relatively minimal technical error, with some experiments (e.g., C. elegans on agar) being more precise than others (e.g., Drosophila basal movement; Fig. 8.37.2A-C). These three setups have worked for our experiments (Houtkooper et al., 2013; Mouchiroud et al., 2013; Williams et al., 2014), but we provide them as a guideline for how to create a simple setup adapted to a particular experiment, rather than as a definitive guide on how to quantify movement.

Any video camera which records at a constant frame rate can be used for tracking, although a video microscope is most likely necessary for $C$. elegans to provide sufficient resolution (Fig. 8.37.1A). High contrast is recommended between the tracked objects and the background. For each experiment, the physical setup of the tracking device and the camera settings (e.g., zoom setting) should be precisely fixed and noted to avoid subtle variations in results from day to day.

For quantitative measurements of $C$. elegans movement on solid agar media, we use a Nikon microscope with a DS-Fi1 camera head connected via a DS-U2 controller to a standard Windows 7 PC by USB (Fig. 8.37.1A). The associated camera software, Nikon NIS Elements, is used to display the microscope image onto the computer and for video tracking (Fig. 8.37.1A). All videos are recorded at $1280 \times 960$ with the objective at the lowest magnification $(0.8 \times)$ for the largest field of view and maximum contrast for distinguishing worms from the plate. Ten worms are placed on $2.5-\mathrm{cm}$ dishes and recorded with bright field for a period of $90 \mathrm{sec}$. Five to ten plates are recorded for each condition. All videos are saved in .avi format at 1 frame per second (fps).

In the three experimental setups (Basic Protocol and Alternate Protocols 1 and 2), we generate two different video formats, three different frame rates, and two different video resolutions. To analyze all videos, we use a single modified version of the Parallel Worm Tracker in MATLAB, in which we have implemented library changes and the addition of the "TransferTracks" step, described below, in order to export all the

Behavioral Neuroscience 
acquired data and use different experimental setups. The original Parallel Worm Tracker (http://wormsense.stanford.edu/tracker/) is a three-step program optimized for tracking C. elegans over arbitrary recording periods, which outputs general movement parameters such as average velocity, turning distance, and location (Ramot et al., 2008). In spite of the three different experimental conditions used, varied recording times, and recording settings, the updated software is capable of analyzing data generated in all three setups using the same algorithms and workflow. Videos for each experiment are placed into individual folders and navigated to MATLAB. The first part of the program, the MovementTracker, processes the videos and generates a track for each individual worm, displaying how its position changes over time. The tracks files are then analyzed with the ObjectAnalyzer to calculate several parameters including the speed, distance, turning, acceleration, and location avoidance; and, any falsely detected tracks may be removed. Finally, the analyzed tracks are summarized with the TransferTracks function, which provides the summary statistics for each measurement such as the average speed, the size of the recorded objects, and the amount each individual turned. The output data may then be quickly analyzed. A step-by-step manual with frequently asked questions is provided in the Supporting Materials File.

\section{Materials}

Nematode growth medium (NGM) agar plates (see recipe) with and without 5 $\mathrm{mg} / \mathrm{ml}$ fluorouracil (5-FU; see recipe)

Bacterial solution: E. coli strains OP50 and HT115

C. elegans

Worm pick made of 30-G platinum wire and glass Pasteur pipet

Stereomicroscope (e.g., Nikon SMZ1000 with Plan Apo 0.5× WD123 objective)

Color digital camera with microscope-computer link (e.g., Nikon DS-Fi1)

Camera control unit (e.g., Nikon DS-L2)

Recording software to make videos from the digitized microscope (e.g.,

NIS-Elements F, neMESYS User Interface, AutoScreenRecorder Pro)

Computer running the following software programs:

MATLAB

The Movement Tracker (available at http://auwerx-lab.epfl.ch/page-130312-en.html)

Microsoft Office Suite

Appropriate graphing software

NOTE: All solutions and equipment coming into contact with $C$. elegans must be sterile, and aseptic technique should be used accordingly.

\section{Synchronization of the worms}

1. Prepare NGM agar plates and bacterial solution following standard procedures and according to the conditions of your experiment (i.e., mutant strains, RNAi, compound treatment).

The commonly used bacterial strains for $C$. elegans feeding and maintenance, OP50 and HT115, are inoculated overnight in LB (see APPENDIX 2A) according to standard procedures (http://www.lifesciences.sourcebioscience.com/clone-products/non-mammalian/celegans/c-elegans-rnai-library/ahringer-lab-clone-information-and-rnai-feeding-proto col) and harvested the following day for seeding on the NGM plates. Bacterial feeding RNAi experiments were carried out as previously described (Kamath et al., 2000) using clone mrps-5 (E02A10.1).

Quantitative

Analysis of

Movement in

Invertebrate Models

Instructions for maintaining $C$. elegans strains can be obtained from The WormBook (http://wbg.wormbook.org/). Briefly, worms are grown at $20^{\circ} \mathrm{C}$ on $\mathrm{NGM}$ plates seeded with OP50 or HT115 bacteria unless otherwise stated. Strains used for these 
protocols were wild-type Bristol N2, AM44 (rmIs190[F25B3.3p::Q67::CFP]), AM725

(rmIs290[unc-54p::Hsa-sod-1(127X)::YFP]), and LS587 (dys-1(cx18) I;hlh-1(cc561)

II).

2. Pretreatment: The day after preparation of NGM plates, use a worm pick to pick L4 worms on these plates.

Pretreatment is only necessary in the case of RNAi or compound treatment that you wish to be started during embryogenesis. If you wish to only measure movements of worm strain-without any compound or RNAi treatment-the pretreatment step is not necessary.

The worm pick is made by placing the platinum wire at the narrow end of a Pasteur pipet and fixing by heat the glass pipet around the wire. The protruding extremity of the wire is flattened using pliers.

Usually 3 worms per plate are enough if the strain develops at a normal rate.

3. Experiment: Three days after pretreatment, pick $10 \mathrm{~L} 4$ worms from the pretreatment plates, and place on fresh NGM agar plates containing 5-FU to avoid progeny development.

A minimum of 5 plates containing 10 worms is recommended to reach a significant number of animals per condition.

Make sure the worms are well synchronized at the L4 stage before beginning the recording, and ensure that the NGM agar plates are not contaminated with any kind of bacteria, fungi, or yeasts.

During a long-term experiment (up to 9 days after the first day of adulthood for the wildtype N2), be sure to remove dead worms before recording. We also recommend adjusting the sample size for such a long-term experiment with a minimum of 10 plates containing 10 worms per condition.

If an experiment includes strains of worms showing a strong defect in mobility, the population can be stimulated by tapping the plates two to three times before the video recording. In addition, to avoid plate contamination or bacterial food source exhaustion, the worms should be moved to new plates every 3 days.

\section{Video recording}

4. Prepare software and the microscope for recording:

a. Turn on the Nikon Digital Sight camera and the power sources of the microscope.

b. Open NIS-Elements F software on the computer.

Once the software is open, you can see the microscope's field of view (FOV).

c. Set the zoom of the microscope to $0.8 \times$ (minimum), and turn the light brightness to maximum.

It is recommended to obtain as uniform a white background as possible to reduce noise in the tracking videos.

d. Set video mode to $1: 1$ and the recording parameters of the NIS-Element $F$ software as follows (fine-tune if required):

Set mode to "Manual Exposure"

Gain $=1.2 \times$

Exposure $=1 / 250 \mathrm{sec}$

Contrast $=$ antireflex.

If a higher video frame rate is desired, these settings may need adjustment.

Behavioral Neuroscience 
5. Open "AutoScreenRecorder Pro" and put your uncovered plate under the microscope for recording. Focus on the worms, and make sure the microscope is set to computer and not binoculars.

6. Recording: Press "Record Now" and, when the recording is done, enter the file name. To facilitate the tracking step, ensure videos are numbered with a sequential number at the end (e.g., Day1_N2_plate1.avi followed by Day1_N2_plate2.avi).

Be sure to use the same recording settings (especially the zoom) for each day of an experiment to ensure consistent results. The default recording length is $90 \mathrm{sec}$. It may be changed, but be careful if doing so, particularly if modifying to record $<45 \mathrm{sec}$.

Begin the recording procedure when worms are adults (1 day after the transfer of the L4), and record videos of the plates every day for 7 or 8 days. Try to have as many worms as possible in the recording field, and do not hesitate to fine-tune the parameters (gain and/or exposure) such that you have the best contrast between the worms and the background. Depending on the bacteria (OP5O or HT115) and the age of the agar plates, these parameters have to be adjusted to obtain a proper white background.

For long-term experiments with multiple time points, videos can be analyzed later when all the videos of experiments have been recorded.

\section{Video analysis}

7. Tracking: Open MATLAB and navigate to the folder in which you have your videos. Type MovementTracker into the command window and press return. In the window that pops up, change the movie name into the title of your videos (without avi extension; Fig. 8.37.3A). Then change the Start and End to whatever your list is.

MovementTracker generates one “.mat” file per video tracked.

The default tracking parameters are (Fig. 8.37.3D):

Min Single Object Size Area: 40 pixels

Max Single Object Size Area: 400 pixels

Max Distance Between Successive Frames: 25 pixels

Max Size Change Between Successive Frames: 100 pixels

Shortest Valid Track: 45 frames

Use Auto Thresholding Correction Factor: 0.05.

All the settings are designed for adult worms. If you wish to measure larval worms, many settings not discussed here must be modified. These parameters might be finetuned (Movement Tracker Preferences; Fig. 8.37.3D) in order to detect as many worms as possible. For example, if your worms are small or at the larval stages, increase the minimum single worm size area; or, if they are moving fast, decrease the shortest valid track and the maximum distance between successive frames. Another option is to increase the value of the auto thresholding factor, which allows detecting more objects even with weak contrast or in the case of smaller worms. However, this will increase the time required for the video analysis.

8. Analysis: When the tracking is done, type ObjectAnalyzer on the MATLAB command window and press return. In the window that opens, click "Tracks" and "Open Tracks File" (Fig. 8.37.3C). Use the scrollbar on the top right of the window to look at each track, and delete the tracks that are not worms by hitting "delete track." After analyzing the tracks, go again under "Tracks" and click on "Analyze All Tracks" to generate a file with all analyzed tracks for each video and named after your original filename but with an additional "a" at the end (e.g., Day1_N2_plate1a.avi).

At the end, you should have the same number of analyzed files as recorded videos.

Quantitative

Analysis of

Movement in

Invertebrate Models

8.37.6 


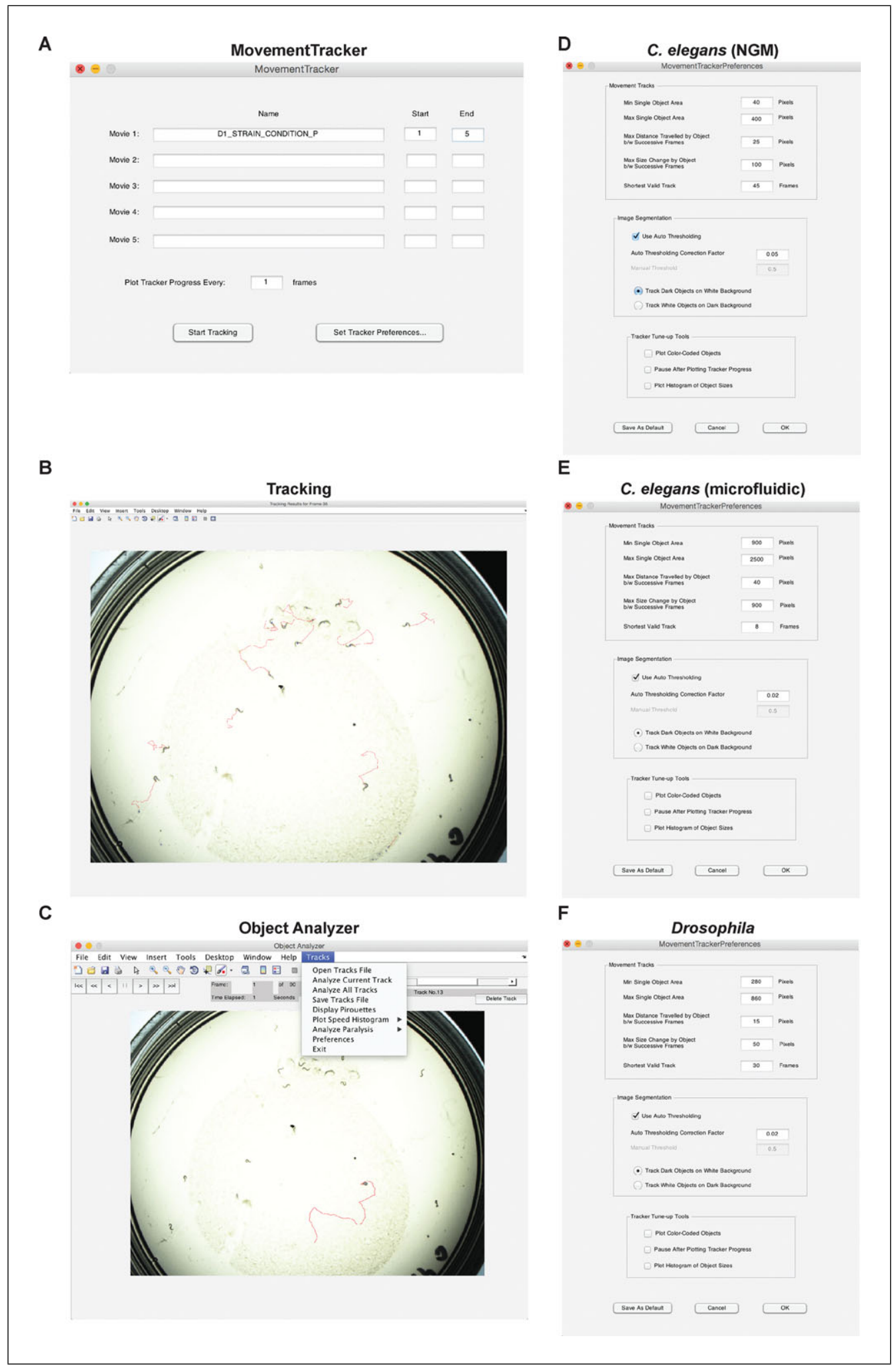

Figure 8.37.3 Workflow of the Movement Tracker program. Representation of the successive sequences of the Movement Tracker, including the Movement Tracker program (A), the tracking step (B), and Object Analyzer program (C) as well as the default tracking parameters for $C$. elegans on solid agar medium (D), for $C$. elegans in a microfluidic chip (E), and for Drosophila (F).

Behavioral Neuroscience 
ALTERNATE PROTOCOL 1
9. Transfer: In order to get a summary of all the data from all the worms, generate an Excel file using a function called "TransferTracks." In the command window of MATLAB, type:

TransferTracks ( \{ 'Day1_N2_plate1a' ; 'Day1_N2_plate2a ' ; '

...' \}, 'Movement_N2_treatment1_Day1.xls')

and press return.

Now, you have an output file called “Movement_N2_compound1_Day1.xls" that contains a summary of all your plates with one row for each worm giving many parameters such as average size, speed, distance, etc.

\section{Data analysis}

10. Open the Excel file that contains all the data from the tracking of the worms, which includes a summary datasheet with information on the individual speed mean, distance, and size of the objects. Calculate for each group the average and the SD of the speed mean, and compute the number of worms you have for each condition.

In addition, for the movement of the nematode on solid media, the function TransferTracks allows you to obtain individual values for speed, angular speed, and direction. This last parameter is particularly interesting as it allows calculation of the number of shifts in direction, which we report here to consistently increase in conditions of impaired mobility in the worm (see Anticipated Results).

If your knockdown or pharmacological treatment has a strong impact on the worm size compared to the control population, you may have to normalize your data according to the size of each individual. For this purpose, the speed mean has to be divided by the mean area value.

11. Plot the graphs with appropriate software.

Examples are shown in Anticipated Results.

\section{ADULT C. elegans ON CHIP}

In liquid media, $C$. elegans have strikingly different movement patterns than on agar plates-in particular, the extremities move substantially more. Thus, several recording parameters are changed, although the same microscope and setup is used but with a stronger magnification $(4 \times)$ and recording at a higher frame rate (at least $4 \mathrm{fps}$ ). As worms may overlap in liquid media, the number of worms is reduced to only ten per chamber to reduce possibilities of path intersection. Thus twice as many chambers must be used to obtain the minimum 50 to 100 worm tracks recommended. Microfluidic chambers can, however, be arranged in high-density matrices, thereby significantly increasing the number of worms that can be analyzed per unit area in a same field of view.

\section{Materials}

Nematode growth medium (NGM) agar plates (see recipe)

$S$ medium (see recipe)

Bacterial solution: E. coli strains OP50 and HT115

C. elegans

Worm pick made of 30-G platinum wire and glass Pasteur pipet 1-ml borosilicate H-TLL-PE syringe (e.g., Innovative Labor Systeme) Microfluidic chip Syringe pump (e.g., Cetoni Nemesys)

Microline ethyl vinyl acetate tubing $(0.51 \mathrm{~mm}$ i.d., $1.52 \mathrm{~mm}$ o.d.; e.g., Fisher Scientific) 
Shut-off fluidic valve (e.g., Upchurch Shut-Off Valve, P-783)

Stereomicroscope (e.g., Nikon SMZ1000 with Plan Apo 0.5× WD123 objective)

Color digital camera with microscope-computer link (e.g., Nikon DS-Fi1)

Camera control unit (e.g., Nikon DS-L2)

Recording software to make videos from the digitized microscope (e.g.,

NIS-Elements F, neMESYS User Interface, AutoScreenRecorder Pro)

Computer running the following software programs:

MATLAB

The Movement Tracker (available at http://auwerx-lab.epfl.ch/page-130312-en.html)

Microsoft Office Suite

Appropriate graphing software

NOTE: All solutions and equipment coming into contact with $C$. elegans must be sterile, and aseptic technique should be used accordingly.

\section{Synchronization of the worms}

1. Prepare nematode growth medium (NGM) agar plates, $\mathrm{S}$ medium, and bacteria solution following standard procedures and according to the conditions of your experiment (e.g., mutant strains, RNAi, compound treatment).

The commonly used bacterial strains for $C$. elegans feeding and maintenance, OP50 and HT115, are inoculated overnight in LB (see APPENDIX 2A) according to standard procedures (http://www.lifesciences.sourcebioscience.com/clone-products/non-mammalian/celegans/c-elegans-rnai-library/ahringer-lab-clone-information-and-rnai-feeding-proto col) and harvested the following day for seeding on the NGM plates. Bacterial feeding RNAi experiments were carried out as previously described (Kamath et al., 2000) using clone mrps-5 (E02A10.1).

Instructions for maintaining $C$. elegans strains can be obtained from The WormBook (http://wbg.wormbook.org/). Briefly, worms are grown at $20^{\circ} \mathrm{C}$ on $\mathrm{NGM}$ plates seeded with OP50 or HT115 bacteria unless otherwise stated. The strain used for this protocol was wild-type Bristol N2.

2. One day after preparation, pick gravid adult worms on these plates.

The worm pick is made by placing the platinum wire at the narrow end of a Pasteur pipet and fixing by heat the glass pipet around the wire. The protruding extremity of the wire is flattened using pliers.

Usually 5 adult worms per plate is enough if the strain develops at a normal rate.

In the case of RNAi or compound treatment that you wish to be started during embryogenesis, the worm population must be pretreated. If you wish to only measure movements of worm strain-without any compound or RNAi treatment-the pretreatment step is not necessary.

3. Three days later, wash the worm culture plate of interest with $500 \mu \mathrm{l} \mathrm{S}$ medium to suspend the whole worm population in liquid.

Use a glass pipet to recover worms as they will stick to plastic.

\section{Preparation of the chip}

4. Fill a syringe with $800 \mu \mathrm{l} \mathrm{S}$ medium, position it into the syringe pump, and connect it to the microfluidic chip inlet via plastic tubing. Connect the shut-off valve to the chip outlet via tubing as well.

Microfluidic chip devices are prepared by soft lithography using 2-layer SU-8 molds. See Cornaglia et al. (2015) for a detailed fabrication protocol. Briefly, 4-in. 550- $\mu \mathrm{m}$ thick Si wafers (Center of MicroNanotechnology of EPFL), GM 1070 SU-8 negative photoresist (Gersteltec), and polydimethylsiloxane (PDMS) Sylgard 184 (Dow Corning)

Behavioral Neuroscience 
are assembled as follows: Conventional photolithography is used to pattern a 35- $\mu \mathrm{m}-$ thick layer of $S U-8$ photoresist on wafers. An 85- $\mu m-$ thick layer of $S U-8$ is then patterned on top of the first one. The silicon mold is diced into $15 \mathrm{~mm} \times 18 \mathrm{~mm}$ microchips, which is inserted at the bottom of an aluminum/polymethylmetacrylate (PMMA) mold for PDMS casting. Then, $1.5 \mathrm{~mm}$ diameter steel pins are used to define lateral connections in the device for external tubing insertion. A liquid PDMS mixture (10:1 base:cross-linker weight ratio) is degassed, injected into the mold, and cured at $100^{\circ} \mathrm{C}$ for $1 \mathrm{hr}$. Upon extraction from the mold, each PDMS chip is bonded by plasma-activation to a 150- $\mu \mathrm{m}$ thick glass coverslip.

5. Inject the $\mathrm{S}$ medium into the chip to complete filling of chip and tubes.

6. Close the outlet valve and inject $10 \mu \mathrm{l} \mathrm{S}$ medium to create overpressure within the device and eliminate any air bubbles from the chip by exploiting the permeability of PDMS to air.

The volume of liquid to be injected at this step strongly depends on the size of the total microfluidic volume to be degassed. For excessive liquid volumes injected, high overpressures may cause chip leaking or unbonding.

7. Unplug the inlet tube from the microfluidic chip, suck $10 \mu 1$ worm suspension (from step 3) into it, reconnect it to the device, and inject $30 \mu \mathrm{l} \mathrm{S}$ medium into the chip at a $500 \mu \mathrm{l} / \mathrm{sec}$ flow rate.

The geometry of the chip (Fig. 8.37.1B) is optimized for directly retaining only adult worms inside the microfluidic chamber, selected by their larger size and better swimming abilities/resistance against the flow. Please refer to Cornaglia et al. (2015) for further details about this specific chip design, modification, and use.

All the adult worms within the inlet tube will be retained inside the chamber. Make sure to work with a known worm concentration (e.g., 5 to 10 worms per $10 \mu \mathrm{l}$ ) to control the number of worms to be analyzed.

While for our experiments we used the microfluidic design reported in Cornaglia et al. (2015) the described protocol can be applied to any PDMS microfluidic device. Liquid volumes and worm concentrations should be adjusted according to the size of the microfluidic chamber in which the worms will be loaded. Worms may be, for instance, injected and tracked directly inside wide microchannels or open chambers if no synchronization of the on-chip population is needed or if one is dealing with a presynchronized population. Simple modifications to the suggested chip geometry can be used instead to tune the size of the worms which will be retained inside the tracking chamber (for details see Cornaglia et al., 2015).

While unplugging and plugging back the inlet tube, ensure that you are not introducing air bubbles within the device. Working with hanging liquid droplets at the tube end may help to ensure this.

\section{Video recording}

8. Prepare software and the microscope for recording:

a. Turn on the Nikon Digital Sight camera and the power sources of the microscope.

b. Open the NIS-Elements F software on the computer.

Once it is open, you can see the microscope's field of view (FOV).

c. Set the zoom of the microscope to $4 \times$ and adjust the light brightness to maximum.

d. Set video mode to $1: 1$ and the recording parameters of the NIS-Element $F$ software as follows for bright field recording (very white background; fine-tune if required):

Set mode to "Manual Exposure"

Gain $=1.2 \times$ 
Exposure $=1 / 250 \mathrm{sec}$

Contrast $=$ Antireflex.

9. Open "AutoScreenRecorder Pro" and put the chip under the microscope for recording. Focus on the worms and make sure the microscope is set to computer and not binoculars.

10. Recording: Press "Record Now" and, when the recording is done, enter the file name. Be sure that videos are numbered with a sequential number at the end to facilitate the tracking step (e.g., Day1_N2_chip1.avi followed by Day1_N2_chip2.avi).

Be sure to use the same recording settings (especially the zoom) every day of an experiment to ensure consistent results. Default recording length is $90 \mathrm{sec}$. It may be changed, but be careful if doing so, particularly if modifying to record $<45 \mathrm{sec}$.

Do not hesitate to fine-tune the parameters (gain and/or exposure) such that you have the best contrast between the worms and the background. Note, however, that through the microfluidic chip, good image contrast is typically much easier to obtain than on agar plates because of the much higher optical transparency of all the materials along the imaging light path.

For long-term experiments with multiple time points, videos can be analyzed later when all the videos of experiments have been recorded.

\section{Video analysis}

11. Tracking: Open MATLAB and navigate to the folder in which you have your videos. Type MovementTracker into the command window and press return. In the window that pops up, change the movie name into the title of your videos (without .avi extension; Fig. 8.37.3A). Then change the Start and End to whatever your list is.

MovementTracker generates one ".mat" file per video tracked.

The default tracking parameters are (Fig. 8.37.3E):

Min Single Object Size Area: 900 pixels

Max Single Object Size Area: 2500 pixels

Max Distance Between Successive Frames: 40 pixels

Max Size Change Between Successive Frames: 900 pixels

Shortest Valid Track: 8 frames

Use Auto Thresholding Correction Factor: 0.02.

These parameters may be fine-tuned (Movement Tracker Preferences) in order to detect as many worms as possible. For example, if your worms are small, increase the minimum single worm size area, or, if they are moving fast, decrease the shortest valid track and the maximum distance between successive frames.

12. Analysis: When the tracking is done, type ObjectAnalyzer on the MATLAB command window and press return, which will open a window with several menu items. Click "Tracks" and "Open Tracks File" (Fig. 8.37.3C). Use the scrollbar on the top right of the window to look at each track, and delete the tracks that are not worms by hitting "delete track." After analyzing the tracks, go again under "Tracks" and click on "Analyze All Tracks." to generate a file with all analyzed tracks for each video and named after your original filename but with an additional "a" at the end (e.g., Day1_N2_chip1a.avi).

At the end, you should have the same number of analyzed files as recorded videos.

13. Transfer: In order to get a summary of all the data from all the worms, generate an Excel file using the function "TransferTracks." In the command window of MATLAB, type

Behavioral Neuroscience

8.37.11 
ALTERNATE PROTOCOL 2

Automated Quantitative Analysis of Movement in Invertebrate Models 8.37.12
TransferTracks ( \{ Day1_N2_chip1a ' ; 'Day1_N2_chip2a ' ; ' ...' \}, 'Movement_N2_treatment1_Day1.xls' )

and press return.

Now, you have an output file called “Movement_N2_compound1_Day1.xls” that contains a summary of all your plates with one row for each worm giving many parameters such as average size, speed, distance, etc.

\section{Data analysis}

14. Open the Excel file that contains all the data from the tracking of the worms. Calculate, for each group the average and the SD of the speed mean, and compute the number of worms you have for each condition.

In this particular setup, it is not possible to obtain information on the directionality of the worms when they move in liquid media.

If your knockdown or pharmacological treatment has a strong impact on worm size, you may have to normalize your data according to the size of each individual. For this purpose, the speed mean has to be divided by the mean area value.

15. Plot the graphs with appropriate software.

Examples are shown in Anticipated Results.

\section{D. melanogaster MOVEMENT}

To record Drosophila, we examine several different conditions. The movement motions and speed between flying movement and walking movement are dramatically different. However, due to the extraordinary difficulty of filming and quantifying Drosophila movement in three dimensions, our systems (and to our knowledge, all other quantitative systems) focus on walking speed (Benzer, 1973). Basal movement of Drosophila can be analyzed with our system. In this setup we place 15 flies in small chambers, which provide sufficient space for walking but not for flying. To measure basal movement, the chambers are set atop a backlit panel in a darkroom. The same setup can be used to measure climbing speed of flies by aligning the camera and the chamber on a flat surface and gently tapping the chamber, which makes it suitable also to evaluate movement in climbing assays (Williams et al., 2014). We use a standard Panasonic DSLR GF1 camera with a Leica $45-\mathrm{mm}$ macro objective, which is set $30 \mathrm{~cm}$ from the fly chamber via the tripod mount. However, nearly any setup will work so long as there is a fixed mount for the camera. In this case, the digital camera is fixed vertically above the chambers. After $5 \mathrm{~min}$ in the chamber, once flies have recovered from the $\mathrm{CO}_{2}$ used to transfer them, flies are recorded for $2 \mathrm{hr}$ at a lower fps to reduce file size and analysis time. To distribute possible effects due to differences in time of day, the flies are distributed such that each condition is evenly represented in each recording set of 15 chambers. Sufficient recordings are performed sequentially to obtain a minimum of 20 flies per condition.

\section{Materials}

Drosophila melanogaster strain w1118 and DGRP strains 707 (stock no. 25200), 335 (stock no. 25183), 427 (stock no. 25193), 358 (stock no. 25185), and 786 (stock no. 25206) available from the Bloomington Drosophila Stock Center at Indiana University (http://flystocks.bio.indiana.edu/)

Cornmeal-agar tube (see recipe)

Incubator

Tracking pad (e.g., BioRad Mini-Protean spacer plates)

Drosophila gassing station with $\mathrm{CO}_{2}$ gas

Tissue paper 
SLR camera on fixed tripod with a macro objective

Computer running the following software programs:

MATLAB

The Movement Tracker (available at

http://auwerx-lab.epfl.ch/page-130312-en.html)

Microsoft Office Suite

Appropriate graphing software

NOTE: The same protocol can be used to assay basal movement or climbing speed.

\section{Synchronization of Drosophila}

1. Transfer adult flies to a fresh cornmeal-agar tube, and put the tube in the incubator.

Drosophila lines are reared on cornmeal-agar medium in standard conditions $\left(25^{\circ} \mathrm{C}\right.$, $12 \mathrm{hr}$ light/dark cycle, 60\% humidity). Instructions for maintaining fly stocks can be found in Greenspan (2004).

2. After 3 days of egg laying, discard the adult flies.

3. The day after, discard the first emerging flies.

4. Collect adults during the next 2 days in a separate tube and allow them to mate.

Because age affects fly motility, it is extremely important that all flies are the same age. In case of RNAi or compound treatment, a delay in fly emergence can appear. Adjust the control cohort accordingly to have flies with similar age.

\section{Video recording}

5. Transfer 10 to 15 flies to the tracking pad using light $\mathrm{CO}_{2}$ gassing, and seal the pad with tissue paper.

Two 1-mm casting glass plates (e.g., BioRad) are assembled together to form a chamber. They are taped together on the sides, and the bottom is covered by Parafilm. A folded tissue is used as a semi-permeable plug allowing air to pass through.

6. Allow the flies to recover from the gassing for a few minutes.

7. Set up the recording station making sure the distance between the pad and the camera is the same for each recording.

The camera should record at 5 to $10 \mathrm{fps}$.

8. Start the camera and lightly tap the pad to the surface to send all the flies to the bottom. Tap the chamber hard every $15 \mathrm{sec}$ during the recording to send all flies to the bottom of the plate. Record for $90 \mathrm{sec}$, or for however many technical replicates of the experiment are desired.

Note that for older flies or flies with movement abnormalities a longer interval than 15 sec may be necessary to allow all flies to reach the top of the chamber.

Distance from camera depends entirely on the camera objective used and the size of the casting plates but should be the same for all experiments. Ideally, the tracked object should just fill as much of the video frame as possible.

Make sure the computer can read the video format of the camera; otherwise, verify that your computer can install the proper codec to read the format. Our package is suitable for Windows XP and Windows 8 supported with a 32- or 64-bit version of MATLAB and Macintosh with a 64-bit version of MATLAB. For long-term experiments with multiple time points, videos can be analyzed later when all the videos of experiments have been recorded.

\section{Video analysis}

9. Transfer the video files from the camera to the computer.

Behavioral Neuroscience 
10. Tracking: Open MATLAB and navigate to the folder in which you have your videos. Type MovementTracker into the command window and press return. In the window that pops up, change the movie name to the title of your videos (without the video format extension; Fig. 8.37.3A). Then change the Start and End to whatever your list is.

MovementTracker generates one ".mat” file per video tracked.

The default tracking parameters are (Fig. 8.37.3F):

Min Single Target Object Size: 280 pixels

Max Single Target Object Size: 860 pixels

Max Distance Between Successive Frames: 15 pixels

Max Size Change Between Successive Frames: 50 pixels

Shortest Valid Track: 30 frames

Use Auto Thresholding Correction Factor: 0.02

These parameters can be fine-tuned (Movement Tracker Preferences) in order to detect all the flies. For example, if your flies are small, increase the minimum single target object size area, or, if they are moving fast, decrease the shortest valid track and the maximum distance between successive frames. These parameters for fly tracking have to be adjusted depending on the video resolution, frame rate, and video contrast. The default parameters shown were created for a video resolution of 720 pixels and a frame rate of $3 \mathrm{fps}$.

11. Analysis: When the tracking is done, type ObjectAnalyzer on the MATLAB command window and press return, which will open a window with several menu items. Click "Tracks" and "Open Tracks File" (Fig. 8.37.3C). Use the scrollbar on the top right of the window to look at each track, and delete the tracks that are not flies by hitting "delete track." After analyzing the tracks, go again under "Tracks" and click on "Analyze All Tracks" to generate a file with all analyzed tracks for each video and named after your original filename but with an additional " $\mathrm{a}$ " at the end, denoting "analyzed" (e.g., Genotype_treatment_repeat1a.avi).

At the end, you should have the same number of analyzed files as of recorded videos.

12. Transfer: In order to get a summary of all the data from all the flies, generate an Excel file using the function called TransferTracks. In the command window of MATLAB, type

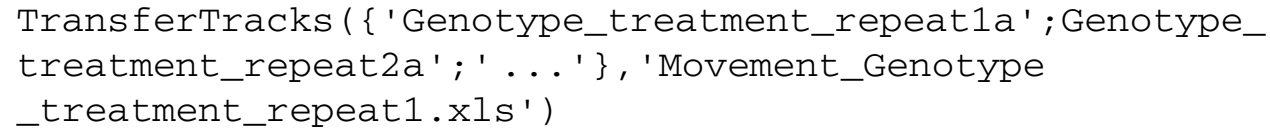

and press return.

Now, you have an output file called "Movement_Genotype_treatment_repeat1.xls" that contains a summary of all your plates with one row for each fly giving many parameters such as average size, speed, distance, etc.

\section{Data analysis}

13. Open the Excel file that contains all the data from the tracking of the flies. Convert the speed to distance by taking the area-under-the-curve (AUC) integral of their velocity. Calculate for each group the average and the SD of the AUC, and compute the number of flies you have for each condition.

If your genotype or pharmacological treatments have a strong impact on fly size, you may have to normalize your data according to the size of each individual. For this purpose, the speed mean has to be divided by the mean area value.

If the flies are treated in such a way that they no longer have the natural tendency to move to the top of a vertical chamber, an alternative experimental setup will be necessary. 
14. Plot the graphs with appropriate software.

Examples are shown in Anticipated Results.

\section{REAGENTS AND SOLUTIONS}

Use deionized, distilled water in all recipes and protocol steps. For common stock solutions, see APPENDIX 2A.

\section{Cornmeal-agar medium}

$6.2 \mathrm{~g}$ agar

58.8 g Farigel (e.g., Westhove N. FMZH1)

$58.8 \mathrm{~g}$ yeast

$100 \mathrm{ml}$ fruit juice

$4.9 \mathrm{ml}$ propionic acid

$26.5 \mathrm{ml}$ Moldex (100 g/liter methyl-4-hydroxybenzoate [e.g., VWR] in 95\% ethanol)

Bring to 1 liter with $\mathrm{H}_{2} \mathrm{O}$

Store at $4{ }^{\circ} \mathrm{C}$ for up to 3 weeks

\section{5-Fluorouracil (5-FU), 5mg/ml}

Add $5 \mathrm{mg}$ 5-FU (Sigma, cat. no. 47576) to $1 \mathrm{ml} \mathrm{H}_{2} \mathrm{O}$. Vortex for several minutes at room temperature. Filter sterilize and store at $4^{\circ} \mathrm{C}$ for up to 3 months.

5-FU is toxic and should be handled in a fume hood.

\section{Nematode growth medium (NGM) plates}

$3 \mathrm{~g} \mathrm{NaCl}$

$17 \mathrm{~g}$ agar

2.5 g Bacto Peptone

$1 \mathrm{ml} 5 \mathrm{mg} / \mathrm{ml}$ cholesterol

$1 \mathrm{ml} 1 \mathrm{M} \mathrm{CaCl}_{2}$

$1 \mathrm{ml} 1 \mathrm{M} \mathrm{MgSO}_{4}$

$25 \mathrm{ml} 1 \mathrm{M} \mathrm{KH}_{2} \mathrm{PO}_{4}, \mathrm{pH} 6$

Bring to 1 liter with $\mathrm{H}_{2} \mathrm{O}$

Filter-sterilize and store at $4^{\circ} \mathrm{C}$ for up to 6 months

For the preparation of NGM and bacteria solution dedicated to RNAi experiments, please refer to http://www.wormbook.org/chapters/www_introreversegenetics/introrever segenetics.html.

\section{Potassium citrate, $1 \mathrm{M}, \mathrm{pH} 6$}

20 g citric acid monohydrate

$293.5 \mathrm{~g}$ tripotassium citrate monohydrate

1 liter $\mathrm{H}_{2} \mathrm{O}$

Sterilize by autoclaving

Store at room temperature for up to 6 months

\section{$S$ basal}

$5.85 \mathrm{~g} \mathrm{NaCl}$

$1 \mathrm{~g} \mathrm{~K}_{2} \mathrm{HPO}_{4}$

$6 \mathrm{~g} \mathrm{KH}_{2} \mathrm{PO}_{4}$

$1 \mathrm{ml} 5 \mathrm{mg} / \mathrm{ml}$ cholesterol

1 liter $\mathrm{H}_{2} \mathrm{O}$ 
Sterilize by autoclaving

Store at room temperature for up to 6 months

\section{$S$ medium}

1 liter $\mathrm{S}$ basal (see recipe)

$10 \mathrm{ml} 1 \mathrm{M}$ potassium citrate (see recipe)

$10 \mathrm{ml}$ trace metals solution (see recipe)

$3 \mathrm{ml} 1 \mathrm{M} \mathrm{CaCl}_{2}$

$3 \mathrm{ml} 1 \mathrm{M} \mathrm{MgSO}_{4}$

Add components using sterile technique

Do not autoclave

Store at room temperature for up to 6 months

\section{Trace metals solution}

\section{$1.86 \mathrm{~g} \mathrm{Na}_{2}$ EDTA \\ $0.69 \mathrm{~g} \mathrm{FeSO}_{4} \cdot 7 \mathrm{H}_{2} \mathrm{O}$ \\ $0.2 \mathrm{~g} \mathrm{MnCl}_{2} \cdot 4 \mathrm{H}_{2} \mathrm{O}$ \\ $0.29 \mathrm{~g} \mathrm{ZnSO}_{4} \cdot 7 \mathrm{H}_{2} \mathrm{O}$ \\ $0.025 \mathrm{~g} \mathrm{CuSO}_{4} \cdot 5 \mathrm{H}_{2} \mathrm{O}$ \\ 1 liter $\mathrm{H}_{2} \mathrm{O}$}

Sterilize by autoclaving

Store in the dark (i.e., in an envelope in tin foil) at room temperature for up to 6 months

\section{COMMENTARY}

\section{Background Information}

Much of our knowledge on the mechanisms of development and aging have stemmed from, or been proven in, the nematode Caenorhabditis elegans (Antebi, 2007) and in Drosophila (Helfand and Rogina, 2003). However, such studies generally gather only limited observable phenotypic information, such as qualitative assessments of reproductive success, development, size, and activity. The nearubiquitous presence of high-quality video recording devices has made gathering quantitative data on these traits feasible for any laboratory, so long as standardized protocols are followed and simple software packages are available. In particular, subtle variations in movement can now be quantified, raising new possibilities for observing the overall phenotypic effects of genetic and environmental factors in both large screening platforms as well as in small, focused studies. For a wide variety of research projects, whether on metabolism, preference/avoidance tests, or aging, accurate measurement of different movement phenotypes represents a critical aspect of data collection.

To address this need, a wide variety of tracking software programs have been developed in recent years. In addition to commercial applications such as PhenoTracker (TSE Systems), dozens of laboratories have devel- oped their own in-house software for tracking specific aspects of movement in model organisms, including C. elegans (Husson et al., 2012), Drosophila (Branson et al., 2009), and mice (York et al., 2013). However, many of these systems were generally designed with a particular setup and experimental design in mind, so establishing these systems in an individual laboratory often requires a steep learning curve and potentially significant investments in hardware and software; few such trackers are geared towards general setups and cross-species usage. Moreover, closedsource commercially available software cannot be adapted to the needs of an individual laboratory. As such, experiments on movement must either conform to a single setup, attempt to merge results from different tracking systems and algorithms, develop their own software, or adapt existing software. In this manuscript, we describe an update to the Parallel Worm Tracker (Ramot et al., 2008), which we now term more generally as the Movement Tracker and which provides: (1) capability to track multiple species in addition to C. elegans, (2) platform independence (Mac, PC, Linux), (3) hardware independence, (4) experimental adaptability, and (5) highthroughput capabilities. We describe here several diverse, simple experimental setups for using this tracking software and demonstrate 
its capacity using a few simple biological paradigms.

\section{Critical Parameters}

\section{Tracking C. elegans movement on solid media}

For the tracking experiments of C. elegans on solid agar (see Basic Protocol), a minimum of 5 plates containing 10 worms is recommended to reach a significant number of animals recorded and analyzed at the end of the experiment. In addition, the worms should be well synchronized before beginning the recording, and it should be ensured that the NGM agar plates are not contaminated with any kind of bacteria, fungi, or yeasts. For the preparation of NGM and bacteria solution dedicated to RNAi experiments, please refer to the WormBook (http://www.wormbook.org/). When working with $C$. elegans, aseptic technique must be used. All glass vials, flasks, media and reagents need to be sterilized before use.

In order to reduce noise in the tracking videos, it is recommended to obtain as uniform a white background as possible. Begin the recording procedure when worms are adults (1 day after the transfer of the L4 worms), and record videos of the plates every day for 7 or 8 days. Try to have as many worms as possible in the recording field, and do not hesitate to fine-tune the parameters (gain and/or exposure) such that you have the best contrast between the worms and the background. Depending on the bacteria (OP50 or HT115) and the age of the agar plates, these parameters have to be adjusted to obtain a proper white background. After video recording and analysis with the Movement Tracker, you should have the same number of analyzed files as recorded videos. Finally, if your knockdown or pharmacological treatment has a strong impact on worm size, you may have to normalize your data according to the size of each individual. For this purpose, the speed mean has to be divided by the mean area value.

\section{Tracking C. elegans movement in liquid media}

For the tracking experiments of C. elegans in microfluidic chip devices (see Alternate Protocol 1), in case of RNAi or compound treatment that you wish to be started during embryogenesis, the worm population has to be pretreated. If you wish to only measure movements of worm strain (without any compound or RNAi treatment) the pretreatment step is not necessary. When manipulating the worms, you must use a glass pipet to recover worms as they will stick to plastic. Also, when working with liquid cultures, make sure to work with a known worm concentration (e.g., 5 to 10 worms per $10 \mu \mathrm{l}$ ) to control the number of worms to be analyzed.

Be sure to use the same recording settings (especially the zoom) every day of an experiment to ensure consistent results. Default recording length is $90 \mathrm{sec}$. It may be changed, but be careful if doing so, particularly if modifying to record $<45 \mathrm{sec}$. Do not hesitate to fine-tune the parameters (gain and/or exposure) such that you have the best contrast between the worms and the background. Note, however, that through the microfluidic chip, good image contrast is typically much easier to obtain than on agar plates because of the much higher optical transparency of all the materials along the imaging light path. Finally, after video recording and analysis with the Movement Tracker, you should have the same number of analyzed files as recorded videos. If your knockdown or pharmacological treatment has a strong impact on worm size, you may have to normalize your data according to the size of each individual. For this purpose, the speed mean has to be divided by the mean area value.

\section{Tracking D. melanogaster movement}

Because age affects fly motility, it is extremely important that all flies are the same age (see Alternate Protocol 2). In the case of RNAi or compound treatment, a delay in fly emergence can appear; therefore, adjust the control cohort accordingly to have flies with similar age. For video acquisition, distance from the camera depends entirely on the camera objective used and the size of the casting plates but should be the same for all experiments. Ideally, the tracked object should just fill as much of the video frame as possible. The parameters for fly tracking have to be adjusted depending in the video resolution, frame rate, and video contrast. The default parameters used in this protocol were created for a video resolution of 720 pixels and a frame rate of $3 \mathrm{fps}$. If your genotype or pharmacological treatments have a strong impact on fly size, you may have to normalize your data according to the size of each individual. For this purpose, the speed mean has to be divided by the mean area value. If the flies are treated in such a way that they no longer have the natural tendency to move to the top of a vertical chamber, an alternative experimental setup will be necessary.
Behavioral Neuroscience 8.37.17

Supplement 77 
Table 8.37.1 Troubleshooting

\begin{tabular}{llll}
\hline Protocol & Problem & Possible cause & Solution \\
\hline $\begin{array}{l}\text { Basic } \\
\text { Protocol }\end{array}$ & Very low mobility of the worms & $\begin{array}{l}\text { Impaired mobility due to aging } \\
\text { or neuromuscular features }\end{array}$ & $\begin{array}{l}\text { Stimulate population by tapping } \\
\text { plates 2-3 times }\end{array}$ \\
& Plate contamination or absence & Contamination by other bacteria & Move worm population to \\
& of bacterial food source on plate & or fungi. Usually bacterial & freshly made and clean NGM \\
& & contaminations will give & plates every 2-3 days, possibly \\
& fast-growing, slimy, & 12 hr before tracking their \\
& dark-colored colonies. Fungal & movement again. This will \\
& contaminations are recognizable & allow worms to not starve or get \\
& by hyphae-based, & contaminated.
\end{tabular}

Recorded tracks are not processed by the software

Low number or no objects detected when of smaller size

Alternate Air bubble appearance within Protocol 1 the microfluidic chip
Introduction of air inside the device during tube unplugging/plugging
In the case of RNAi or compound treatment, flies could show developmental delays

The codecs for reading the camera videos are not installed
Adjust software setup to analyze tracks with $<90$ frames

Adjust the microscope exposure parameters (i.e., brightness, gain); increase the minimum worm size area; increase the auto thresholding factor

While plugging the tube back to the chip, make sure to have a liquid droplet hanging at the tube end. If necessary, perform step 6 again to eliminate any air bubbles which entered the device.

Adjust the control cohort accordingly to have flies with similar ages

Make sure the computer can read the video format of the camera; otherwise, verify that your computer can install the proper codec to read the format recognized or not processed by the software

$\begin{array}{ll}\text { Alternate } & \begin{array}{l}\text { Flies under different treatments } \\ \text { Protocol } 2\end{array} \\ & \begin{array}{l}\text { show a delay in emergence } \\ \text { compared to the control } \\ \text { condition }\end{array} \\ & \begin{array}{l}\text { Recorded tracks are not } \\ \text { recognized or not processed by } \\ \text { the software }\end{array}\end{array}$

Smaller worms (larvae or treated worms) generate less
contrast during visualization

\author{
Stimulate population by tapping
}

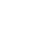

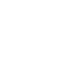




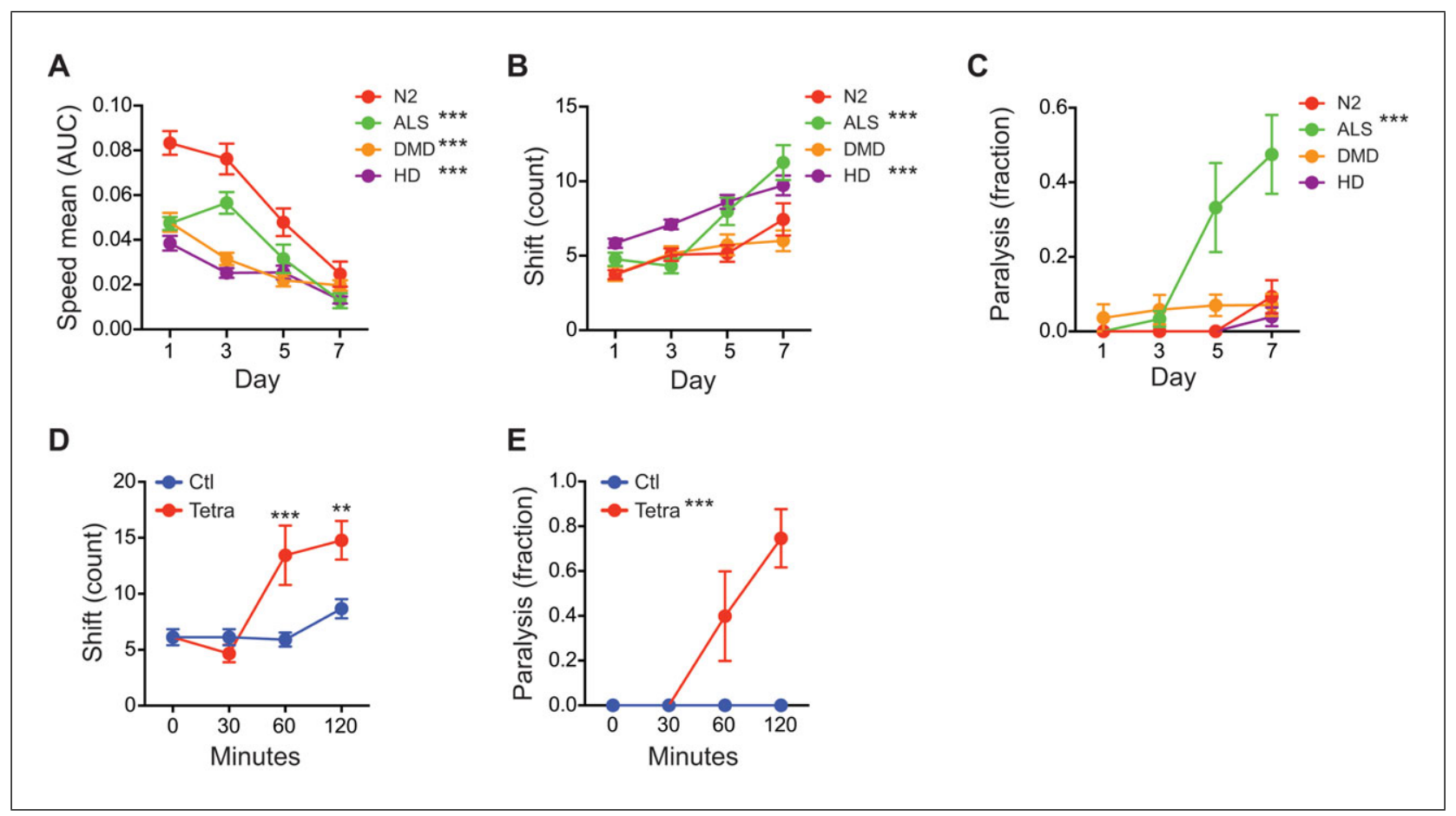

Figure 8.37.4 Movement data obtained in $C$. elegans with neurodegenerative or neuromuscular disease models or pharmacological treatments on solid agar medium. (A) C. elegans models of amyotrophic lateral sclerosis (ALS; AM725), Duchenne muscular dystrophy (DMD; LS587), and Huntington's disease (HD; AM44) significantly reduce mobility compared to the wild-type (N2) population. (B) ALS and HD models, but not the DMD model, increase the number of directional shifts compared to the control. (C) The percentage of paralyzed worms increases significantly over time in the ALS model, but not in the HD and DMD models, compared to the control. Tetramisole (Tetra) treatment $(0.2 \mathrm{mM})$ quickly increases the number of directional shifts (D) and the percentage of paralyzed worms (E) compared to the untreated population (Ctl). Values are mean \pm SEM. ${ }^{* *} P<0.01 ;{ }^{* *} P<0.001$. AUC, area under the curve.

parameter can be used to determine the total number of shifts of direction in the worm population. This parameter consistently increases with aging in all worm strains and is further increased by conditions of neurodegeneration (Fig. 8.37.4B) or by treatments aimed at affecting neurotransmission (Fig 8.37.4D). The individual speed values allow calculation of the percentage of paralyzed worms within the same population or for the same treatment by setting an arbitrary minimum speed threshold (Fig. 8.37.4C and E) as previously reported (Ramot et al., 2008).

\section{Detection of movement changes and improvements in different models and environments}

We have previously shown that treating worms with the antibiotic doxycycline, which affects mitochondrial function by reducing mitochondrial translation, can extend nematode lifespan and improve its fitness (Houtkooper et al., 2013). Similar results have been obtained with genetic interventions, such as reduction in the expression of the gene mrps5 , which results in decreased mitochondrial function and improved fitness (Houtkooper et al., 2013). The Movement Tracker allows reproducible detection of the increased movement of a worm population treated with reduced mrps-5 expression at different ages or doxycycline in different environments such as solid media (NGM plates) or liquid media (microfluidics systems; Fig. 8.37.5A-D). Importantly, the information provided by the software on the size of the tracked objects allows normalization of measured speed values; therefore it is possible to quantify movement changes during treatments affecting the body size of the model organism used (Fig. 8.37.5AC). In addition, it is possible to readily measure variation in the movement of different $D$. melanogaster strains, such as in the DGRP fly population (Fig. 8.37.5E; Mackay et al., 2012).

\section{Time Considerations}

\section{Basic Protocol (adult C. elegans on solid agar media)}

Reagent setup typically takes 1 to 2 days. Steps 1 through 3 take 6 days; steps 4 through 6 can take up to $10 \mathrm{~min}$, depending on the number of conditions included in the experiment. Basically, the video recording of one
Behavioral Neuroscience 


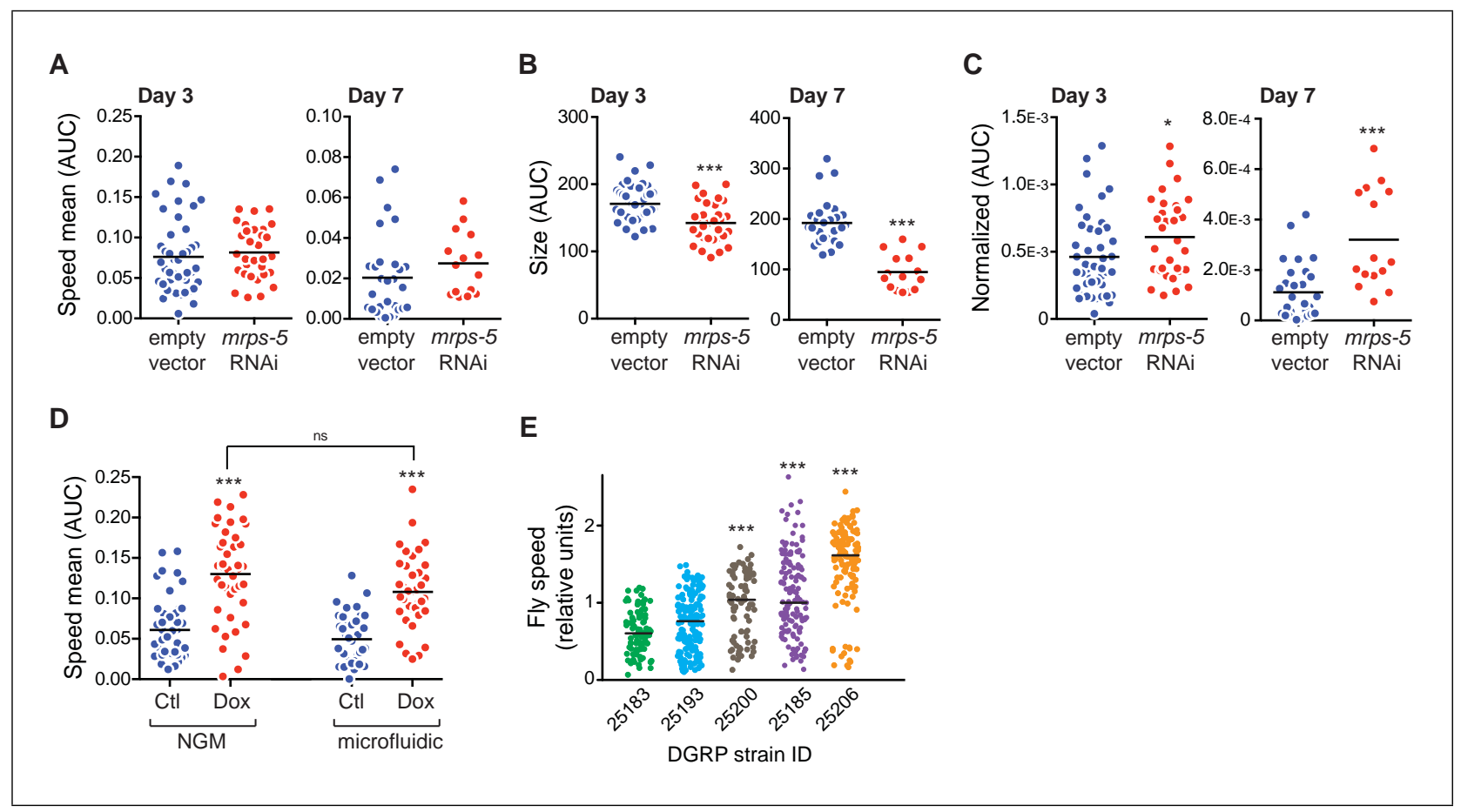

Figure 8.37.5 Movement data obtained in a microfluidic environment and with Drosophila populations. (A-C) mrps-5 RNAi significantly increases the wild-type N2 mobility only when the SpeedMean value is normalized to the size of each individual in young (day 3 of adulthood) and old (day 7 of adulthood) animals. (D) Doxycycline (Dox) treatment (15 $\mu \mathrm{g} / \mathrm{ml})$ significantly increases the movement of worms at D1, either on solid NGM plates or in a microfluidic chip, compared to untreated worms (Ctl). (E) Movement of various DGRP lines with differential expression for the gene spineless. Values are mean \pm SEM. ${ }^{*} P<0.01 ;{ }^{* \star *} P<0.001$. AUC, area under the curve.

condition including 5 plates takes 7 to $8 \mathrm{~min}$. Steps 7 through 9 can take up to $30 \mathrm{~min}$, depending on the number of conditions included in the experiment. Steps 10 and 11 can take up to $1 \mathrm{hr}$.

\section{Alternate Protocol 1 (adult C. elegans on chip)}

Reagent setup typically takes 1 to 2 days for the NGM plates, S-medium, and bacteria plates, 2 days for the mold preparation, and $3 \mathrm{hr}$ for the PDMS-chip preparation. Steps 1 through 3 take 5 days, and steps 4 through 7, 1 hr. Steps 8 through 10 can take up to $10 \mathrm{~min}$, depending on the number of conditions included in the experiment. Basically, the video recording of one condition including 5 chambers takes 7 to $8 \mathrm{~min}$. Steps 11 through 13 can take up to $30 \mathrm{~min}$, depending on the number of conditions included in the experiment, while steps 14 and 15 can take up to $1 \mathrm{hr}$.

\section{Alternate Protocol 2 (D. melanogaster movement)}

Reagent setup typically takes $30 \mathrm{~min}$. Steps 1 through 4 require raising flies. Use standard food to raise flies. Constructing a pad could take up to $30 \mathrm{~min}$. Steps 5 through 8 can take up to $10 \mathrm{~min}$, depending on the number of con- ditions included in the experiment. The video recording of one condition takes around 90 sec, which includes 6 technical replicate runs. Steps 9 through 12 can take up to $30 \mathrm{~min}$, depending on the number of conditions included in the experiment. Steps 13 and 14 can take up to $1 \mathrm{hr}$.

\section{Supporting Materials}

The supporting material file discussed in this article can only be accessed from the online version of this article.

\section{Acknowledgments}

We would like to thank Dr. D. Ramot for developing the Parallel Worm Tracker and providing it open source for the community, Prof. M. Goodman for allowing us to adapt and update the software, and Hao $\mathrm{Li}$ for additional insight on the adaptation of the software. J.A. is the Nestlé Chair in Energy Metabolism, and his research is supported by the EPFL, the NIH (R01AG043930), Systems X (SySX.ch 2013/153), and the SNSF (31003 A-140780). Work in the M.G. laboratory was supported by the EPFL and the EU Ideas program (ERC-2012-AdG-320404). B.D. is supported by the EPFL and Systems X (SySX.ch 2013/153). 


\section{Literature Cited}

Antebi, A. 2007. Genetics of aging in Caenorhabditis elegans. PLoS Genet. 3:1565-1571. doi: 10.1371/journal.pgen.0030129.

Benzer, S. 1973. Genetic dissection of behavior. Sci. Am. 229:24-37. doi: 10.1038/scientificamerican1273-24.

Branson, K., Robie, A.A., Bender, J., Perona, P., and Dickinson, M.H. 2009. High-throughput ethomics in large groups of Drosophila. Nat. Methods 6:451-457. doi: 10.1038/nmeth. 1328.

Cornaglia, M., Mouchiroud, L., Marette, A., Narasimhan, S., Lehnert, T., Jovaisaite, V., Auwerx, J., and Gijs, M.A. 2015. An automated microfluidic platform for C. elegans embryo arraying, phenotyping, and long-term live imaging. Sci. Rep. 5:10192. doi: 10.1038/srep10192.

Greenspan, R.J. 2004. Fly Pushing: The Theory and Practice of Drosophila Genetics, 2nd ed. Cold Spring Harbor Laboratory Press. Cold Spring Harbor, New York.

Helfand, S.L. and Rogina, B. 2003. Genetics of aging in the fruit fly, Drosophila melanogaster. Annu. Rev. Genet. 37:329-348. doi: 10.1146/annurev.genet.37.040103.095211.

Houtkooper, R.H., Mouchiroud, L., Ryu, D., Moullan, N., Katsyuba, E., Knott, G., Williams, R.W., and Auwerx, J. 2013. Mitonuclear protein imbalance as a conserved longevity mechanism. Nature 497:451-457. doi: 10.1038/nature12188.

Husson, S.J., Costa, W.S., Schmitt, C., and Gottschalk, A. 2012. Keeping track of worm trackers. WormBook 22:1-17. doi: 10.1895/ wormbook.1.156.1.

Kamath, R.S., Martinez-Campos, M., Zipperlan, P., Fraser, A.G., and Ahringer, J. 2000. Effectiveness of specific RNA-mediated interference through ingested double-stranded RNA in Caenorhabditis elegans. Genome Biol. 2:research0002.1. doi: 10.1186/gb-20002-1-research0002.

Mackay, T.F., Richards, S., Stone, E.A., Barbadilla, A., Ayroles, J.F., Zhu, D., Casillas, S., Han,
Y., Magwire, M.M., Cridland, J.M., Richardson, M.F., Anholt, R.R., Barron, M., Bess, C., Blankenburg, K.P., Carbone, M.A., Castellano, D., Chaboub, L., Duncan, L., Harris, Z., Javaid, M., Jayaseelan, J.C., Jhangiani, S.N., Jordan, K.W., Lara, F., Lawrence, F., Lee, S.L., Librado, P., Linheiro, R.S., Lyman, R.F., Mackey, A.J., Munidasa, M., Muzny, D.M., Nazareth, L., Newsham, I., Perales, L., Pu, L.L., Qu, C., Ramia, M., Reid, J.G., Rollmann, S.M., Rozas, J., Saada, N., Turlapati, L., Worley, K.C., Wu, Y.Q., Yamamoto, A., Zhu, Y., Bergman, C.M., Thornton, K.R., Mittelman, D., and Gibbs, R.A. 2012. The Drosophila melanogaster Genetic Reference Panel. Nature 482:173-178. doi: 10.1038/nature 10811 .

Mouchiroud, L., Houtkooper, R.H., Moullan, N., Katsyuba, E., Ryu, D., Canto, C., Mottis, A., Jo, Y.S., Viswanathan, M., Schoonjans, K., Guarente, L., and Auwerx, J. 2013. The $\mathrm{NAD}(+) /$ sirtuin pathway modulates longevity through activation of mitochondrial UPR and FOXO signaling. Cell 154:430-441. doi: 10.1016/j.cell.2013.06.016.

Ramot, D., Johnson, B.E., Berry, T.L., Jr., Carnell, L., and Goodman, M.B. 2008. The Parallel Worm Tracker: A platform for measuring average speed and drug-induced paralysis in nematodes. PloS One 3:e2208. doi: 10.1371/journal.pone. 0002208 .

Williams, E.G., Mouchiroud, L., Frochaux, M., Pandey, A., Andreux, P.A., Deplancke, B., and Auwerx, J. 2014. An evolutionarily conserved role for the aryl hydrocarbon receptor in the regulation of movement. PLoS Genet. 10:e1004673. doi: 10.1371/journal.pgen.1004673.

York, J.M., Blevins, N.A., McNeil, L.K., and Freund, G.G. 2013. Mouse short- and longterm locomotor activity analyzed by video tracking software. J. Vis. Exp. 76:50252. doi: $10.3791 / 50252$.

\section{Internet Resources}

http://auwerx-lab.epfl.ch/page-130312-en.html The Movement Tracker software and manual.
Behavioral Neuroscience

8.37.21 